Invest. pens. crit. (ISSN 1812-3864)

Vol. 6, No. 1, enero-abril 2018

pp. 36-50

\title{
Determinación de Necesidades de Capacitación, según Colaboradores y Gerentes de Empresas en la Ciudad de Panamá
}

\author{
Vicente Herrera ${ }^{1 *}$, Miroslava de Zarate ${ }^{1}$, Yolanda Portillo ${ }^{1}$, \\ José $\mathrm{Chu}^{1}$, Carmen Guerra ${ }^{1}$ \\ ${ }^{1}$ Docentes de la Facultad de Negocios de la Universidad Santa María La Antigua de Panamá, \\ * Autor para correspondencia, correo electrónico: drvicenteherrera@gmail.com
}

Recibido: 30 de diciembre de 2017

Aceptado: 22 de marzo de 2018

\begin{abstract}
This research paper describes the attitude of employees and business managers about training needs and other aspects related to training. It is a descriptive and explanatory research, where a questionnaire was applied to a total of 664 collaborators who were visited in the City of Panama, and 146 questionnaires were applied to company managers.

The fieldwork with the collaborators was carried out by undergraduate students, under the guidance of teachers. On the other hand, managers were surveyed by postgraduate students, also under the guidance of USMA faculty. The data was tabulated and processed using the SPSS 24 statistical program. In order to gather the information, a validated questionnaire was applied, which sought the opinions of collaborators and managers on the needs of training courses, and other aspects of training in companies. This research is a contribution of the USMA to show the reality of business training needs, in order to adapt a training offer to the needs of the work environment.
\end{abstract}

Key words: Training, employees, managers, training needs.

\section{Resumen}

El presente trabajo describe las actitudes de los colaboradores y gerentes de empresas sobre las necesidades de capacitación y otros aspectos relacionados con la formación. Es una investigación de tipo descriptiva y explicativa, y donde se aplicó un cuestionario a un total de 664 colaboradores que se visitaron en la Ciudad de Panamá, y se aplicaron 146 cuestionarios a gerentes de empresas.

El trabajo de campo con los colaboradores, fue realizado por estudiantes de licenciatura, bajo la orientación de docentes. Por otro lado, los gerentes fueron encuestados por estudiantes de postgrados, igualmente bajo la orientación de docentes de la USMA. Los datos se tabularon y se procesaron utilizando el programa de estadística SPSS 24. Para reunir la información se aplicó un cuestionario que fuer validado, y que buscaba conocer las opiniones de colaboradores y gerentes sobre las necesidades de cursos de capacitación, y otros aspectos de formación en las empresas. Esta investigación es un aporte de la USMA al mostrar la realidad de las necesidades de 
Invest. pens. crit. (ISSN 1812-3864)

Vol. 6, No. 1, enero-abril 2018

pp. $36-50$

capacitación empresarial, con el fin de adecuar una oferta de capacitación a las necesidades del entorno empresarial.

Palabras clave: Capacitación, colaboradores, gerentes, necesidades de capacitación.

\section{Introducion}

La Facultad de Negocios de la USMA, con el deseo de continuar apoyando a la gestión de la Dirección de Educación Continua, en la función de extensión, establece realizar una investigación que ayude a la toma de decisiones en materia de formación y capacitación empresarial.

La Dirección de Educación Continua representa una modalidad educativa flexible en sus programas, contenidos y métodos, innovada y recreada continuamente de acuerdo con las necesidades de los diversos sectores. Constituye una opción para las personas que, con o sin título profesional, puedan acceder a nuevos y relevantes conocimientos para hacer frente a los procesos de innovación tecnológica y de producción para un mejor desempeño laboral.

La presente investigación, busca conocer las opiniones y necesidades de los colaboradores y gerentes de las empresas, para que la USMA, pueda ofrecer alternativas de educación y, en las diferentes áreas del conocimiento, con Modalidades Educativas convencionales y no convencionales (virtuales), permitiendo así el desarrollo de los colaboradores y gerentes de las empresas. Se estableció como problema de investigación, lo siguiente: ¿Qué necesidades de capacitación tiene los colaboradores y gerentes en las empresas de la Ciudad de Panamá?

En muchas organizaciones hay quienes consideran la capacitación como un gasto innecesario y no como una inversión que beneficiará tanto a la empresa como a sus colaboradores. Es necesario indicar que la capacitación debe ser un proceso continuo porque la realidad, la tecnología y los conocimientos no cesan de cambiar y de desarrollarse. Nada es estático en este mundo. Un profesional o un técnico requerirán actualizaciones en forma periódica porque de lo contrario llegará un momento en que se desactualizan, y no aportan a la organización, y hacen que esta pierda competitividad y productividad.

La capacitación es una de las inversiones más rentables que puede realizar una empresa. Una planificación adecuada del componente humano en una organización capacitar al personal a su cargo. Así, los equipos de trabajo mejorarán su desempeño.

También beneficia a las organizaciones, ya que:

- Mejora la imagen de la organización.

- Mejora la relación jefes - subordinados.

- Coadyuva a la comprensión y adopción de políticas.

- Ayuda a reducir los costos.

- En el campo individual, la capacitación presenta beneficios como los siguientes:

- Ayuda a las personas a resolver problemas y a tomar decisiones.

- Formar líderes y mejora las aptitudes comunicativas de las personas.

- Permite el logro de metas individuales. 
Invest. pens. crit. (ISSN 1812-3864)

Vol. 6, No. 1, enero-abril 2018

pp. $36-50$

- Aumenta la confianza en sí mismo, eliminando los temores a la incompetencia o a la ignorancia individual.

\section{Métodos}

Esta investigación es de tipo descriptiva y de diseño no experimental del tipo transversal. La investigación busca evaluar las opiniones de los colaboradores y gerentes de las empresas en Panamá. Se ha considerado como elemento de la población, los colaboradores y gerentes. Para el caso de ellos no tenesmos las cantidades con exactitud, por lo cual decimos que es infinita por lo tanto se hace un cálculo de muestra para población infinita.

Para la muestra de colaboradores se utilizó una fórmula para poblaciones infinitas.

Los parámetros son:

$Z^{2}=1,96$ (si la seguridad es de $95 \%$ )

$\mathrm{p}=$ proporción esperada (en este caso $50 \%=0,5)$

$\mathrm{q}=1-\mathrm{p}$ (en este caso $1-0,5=0,5)$

$\mathrm{e}=$ Error (en este caso se desea $5 \%$ )

La fórmula para población infinita es:

$\frac{\mathrm{Z}^{2} * \mathrm{P} * \mathrm{Q}}{\mathrm{e}^{\underline{2}}}$

Se reemplazan los datos:

$\mathrm{n}=(1.96)^{2}(.5) *(.5)$

$(.05)^{2}$

La muestra es de: 384 personas

En este estudio se aplicaron a colaboradores, un total de 664 encuestas con lo cual se cumplen con creces la cantidad mínima. Para los gerentes se escogieron una cantidad de 146 gerentes. Se utilizo un cuestionario para colaboradores de aproximadamente 19 preguntas, esas preguntas eran de tipo cerradas, pero algunas tenían subpreguntas. Para los Gerentes se elaboró un cuestionario de 22 preguntas en su mayoría cerradas. Las encuestas fueron aplicadas en los meses de noviembre, diciembre de 2016, y en enero, y febrero de 2017.

Los cuestionarios fueron aplicados por un grupo de estudiantes de la USMA, los cuales cursaqban los cursos de Investigación de Mercado, Servicio al cliente, y Metodología de investigación. Los estudiantes de licenciaturas aplicaron a los Colaboradores, y los estudiantes de postgrados a los Gerentes. Se procesaron los datos utilizando el Programa SPSS 24. El Statistical Package for the 
Invest. pens. crit. (ISSN 1812-3864)

Vol. 6, No. 1, enero-abril 2018

pp. $36-50$

Social Sciences (SPSS 24), es un programa estadístico informático muy usado en las ciencias sociales y las empresas de investigación de mercado.

\section{Resultados}

En cuanto a los colaboradores, podemos ver en la Tabla No.1 que un 52.2\% son del sexo femenino, y un $47.8 \%$ son del sexo masculino. Un $45.6 \%$ trabajan en empresas de servicio, seguido por un $28.8 \%$ que trabajan en empresas de comercio, un 14,2\% trabajan en industrias, y en menores cantidades en otras empresas.

Tabla No.1

Actividad de la empresa y sexo de los colaboradores

\begin{tabular}{|l|l|r|r|r|}
\hline \multirow{2}{*}{ Actividad de la Empresa } & \multicolumn{2}{|c|}{ Sexo del encuestado } & \multirow{2}{*}{ Total } \\
\cline { 2 - 5 } & Masculino & Femenino & \multicolumn{1}{|c|}{ Tota } \\
\hline \multirow{2}{*}{ Servicio } & Recuento & 128 & 162 & 290 \\
\cline { 2 - 5 } & $\%$ del total & $20.1 \%$ & $25.5 \%$ & $45.6 \%$ \\
\hline \multirow{2}{*}{ Comercio } & Recuento & 101 & 82 & 183 \\
\cline { 2 - 5 } & $\%$ del total & $15.9 \%$ & $12.9 \%$ & $28.8 \%$ \\
\hline \multirow{2}{*}{ Andustria } & Recuento & 44 & 46 & 90 \\
\cline { 2 - 5 } & $\%$ del total & $6.9 \%$ & $7.2 \%$ & $14.2 \%$ \\
\cline { 2 - 5 } & Recuento & 10 & 5 & 15 \\
\hline Otras & Recuento & $1.6 \%$ & $0.8 \%$ & $2.4 \%$ \\
\cline { 2 - 5 } & $\%$ del total & $3.3 \%$ & $5.8 \%$ & $9.1 \%$ \\
\hline Total & Recuento & 304 & 332 & 636 \\
\cline { 2 - 5 } & $\%$ del total & $47.8 \%$ & $52.2 \%$ & $100.0 \%$ \\
\hline
\end{tabular}

En cuanto a los gerentes, podemos ver en la Tabla No. 2 que un 51.4\% son del sexo masculino, y un $48.6 \%$ son del sexo masculino. Un $41.1 \%$ laboran en empresas de servicio, seguido por un $28.1 \%$ que laboran en empresas de comercio, un $13.0 \%$ laboran en industrias, y en menores cantidades en otras empresas.

Tabla No.2

Actividad de la empresa y sexo de los gerentes

\begin{tabular}{|l|l|r|r|r|}
\hline \multirow{2}{*}{ Actividad de la Empresa } & \multicolumn{2}{|c|}{ Sexo del encuestado } & \multirow{2}{*}{ Total } \\
\cline { 3 - 4 } & Masculino & Femenino & \multicolumn{1}{|c|}{ Torio } \\
\hline \multirow{2}{*}{ Servicio } & Recuento & 31 & 29 & 60 \\
\cline { 2 - 5 } & $\%$ del total & $21.2 \%$ & $19.9 \%$ & $41.1 \%$ \\
\hline \multirow{2}{*}{ Comercio } & Recuento & 20 & 21 & 41 \\
\cline { 2 - 5 } & $\%$ del total & $13.7 \%$ & $14.4 \%$ & $28.1 \%$ \\
\hline Industria & Recuento & 9 & 10 & 19 \\
\cline { 2 - 5 }
\end{tabular}


Invest. pens. crit. (ISSN 1812-3864)

Vol. 6, No. 1, enero-abril 2018

pp. $36-50$

\begin{tabular}{|l|l|r|r|r|}
\hline \multirow{3}{*}{ Agropecuarias } & $\%$ del total & $6.2 \%$ & $6.8 \%$ & $13.0 \%$ \\
\cline { 2 - 5 } & Recuento & 4 & 0 & 4 \\
\cline { 2 - 5 } & $\%$ del total & $2.7 \%$ & $0.0 \%$ & $2.7 \%$ \\
\hline \multirow{3}{*}{ Otras } & Recuento & 11 & 11 & 22 \\
\cline { 2 - 5 } & $\%$ del total & $7.5 \%$ & $7.5 \%$ & $15.1 \%$ \\
\hline \multirow{2}{*}{ Total } & Recuento & 75 & 71 & 146 \\
\cline { 2 - 5 } & $\%$ del total & $51.4 \%$ & $48.6 \%$ & $100.0 \%$ \\
\hline
\end{tabular}

En cuanto a los colaboradores, podemos ver en la Tabla No. 3 que un 39.3\% opinan que es más urgente capacitar al personal administrativo, y un 29.6\% consideran que es al personal operativo. En todos los tipos de empresas consideran que es prioritario capacitar al personal administrativo.

Tabla No.3

Area prioritaria a capacitar por tipo de actividad de la empresa, según los colaboradores

\begin{tabular}{|c|c|c|c|c|c|c|c|}
\hline \multirow{2}{*}{\multicolumn{2}{|c|}{ Actividad de la Empresa }} & \multicolumn{5}{|c|}{ Área de la empresa } & \multirow{3}{*}{$\frac{\text { Total }}{290}$} \\
\hline & & \multirow{2}{*}{$\begin{array}{r}\text { Operativo } \\
92\end{array}$} & \multirow{2}{*}{$\begin{array}{r}\text { Administrativo } \\
115\end{array}$} & \multirow{2}{*}{$\frac{\text { Técnico }}{26}$} & \multirow{2}{*}{$\frac{\text { Supervisión }}{28}$} & \multirow{2}{*}{$\begin{array}{r}\text { Ejecutivo } \\
29\end{array}$} & \\
\hline Servicio & Recuento & & & & & & \\
\hline & $\%$ del total & $14.5 \%$ & $18.1 \%$ & $4.1 \%$ & $4.4 \%$ & $4.6 \%$ & $45.6 \%$ \\
\hline \multirow[t]{2}{*}{ Comercio } & Recuento & 47 & 73 & 9 & 17 & 37 & 183 \\
\hline & $\%$ del total & $7.4 \%$ & $11.5 \%$ & $1.4 \%$ & $2.7 \%$ & $5.8 \%$ & $28.8 \%$ \\
\hline \multirow[t]{2}{*}{ Industria } & Recuento & 25 & 30 & 12 & 13 & 10 & 90 \\
\hline & $\%$ del total & $3.9 \%$ & $4.7 \%$ & $1.9 \%$ & $2.0 \%$ & $1.6 \%$ & $14.2 \%$ \\
\hline \multirow[t]{2}{*}{ Agropecuarias } & Recuento & 5 & 5 & 1 & 3 & 1 & 15 \\
\hline & $\%$ del total & $0.8 \%$ & $0.8 \%$ & $0.2 \%$ & $0.5 \%$ & $0.2 \%$ & $2.4 \%$ \\
\hline \multirow[t]{2}{*}{ Otras } & Recuento & 19 & 27 & 4 & 3 & 5 & 58 \\
\hline & $\%$ del total & $3.0 \%$ & $4.2 \%$ & $0.6 \%$ & $0.5 \%$ & $0.8 \%$ & $9.1 \%$ \\
\hline \multirow[t]{2}{*}{ Total } & Recuento & 188 & 250 & 52 & 64 & 82 & 636 \\
\hline & $\%$ del total & $29.6 \%$ & $39.3 \%$ & $8.2 \%$ & $10.1 \%$ & $12.9 \%$ & $100.0 \%$ \\
\hline
\end{tabular}

En cuanto a la opnión de los gerentes, podemos ver en la Tabla No. 4, que un 31.5\% opinan que es más urgente capacitar al personal operativo, y un 29.5\% consideran que es al personal administrativo, y los de las industrias piensan que son el personal operativo. Los gerentes de empresas de servicio, opinan que es más urgente capacitar al personal operativo, y los gerentes de empresas de comercio, piensan que más urgente es capacitar al personal administrativo. 
Invest. pens. crit. (ISSN 1812-3864)

Vol. 6, No. 1, enero-abril 2018

pp. $36-50$

\section{Tabla No.4}

Area prioritaria a capacitar por tipo de actividad de la empresa, según los gerentes

\begin{tabular}{|c|c|c|c|c|c|c|c|}
\hline & & \multicolumn{5}{|c|}{ Nivel de la empresa que requiere capacitación } & \multirow[b]{2}{*}{ Total } \\
\hline \multicolumn{2}{|c|}{ Actividad de la Empresa } & Operativo & Administrativos & Técnicos & Supervisores & $\begin{array}{l}\text { Ejecutivos } \\
\text { gerenciales }\end{array}$ & \\
\hline \multirow[t]{2}{*}{ Servicio } & Recuento & 22 & 12 & 10 & 8 & 8 & 60 \\
\hline & $\%$ del total & $15.1 \%$ & $8.2 \%$ & $6.8 \%$ & $5.5 \%$ & $5.5 \%$ & $41.1 \%$ \\
\hline \multirow[t]{2}{*}{ Comercio } & Recuento & 10 & 19 & 3 & 4 & 5 & 41 \\
\hline & $\%$ del total & $6.8 \%$ & $13.0 \%$ & $2.1 \%$ & $2.7 \%$ & $3.4 \%$ & $28.1 \%$ \\
\hline \multirow[t]{2}{*}{ Industria } & Recuento & 6 & 5 & 5 & 2 & 1 & 19 \\
\hline & $\%$ del total & $4.1 \%$ & $3.4 \%$ & $3.4 \%$ & $1.4 \%$ & $0.7 \%$ & $13.0 \%$ \\
\hline \multirow[t]{2}{*}{ Agropecuarias } & Recuento & 1 & 2 & 0 & 1 & 0 & 4 \\
\hline & $\%$ del total & $0.7 \%$ & $1.4 \%$ & $0.0 \%$ & $0.7 \%$ & $0.0 \%$ & $2.7 \%$ \\
\hline \multirow[t]{2}{*}{ Otras } & Recuento & 7 & 5 & 7 & 3 & 0 & 22 \\
\hline & $\%$ del total & $4.8 \%$ & $3.4 \%$ & $4.8 \%$ & $2.1 \%$ & $0.0 \%$ & $15.1 \%$ \\
\hline \multirow[t]{2}{*}{ Total } & Recuento & 46 & 43 & 25 & 18 & 14 & 146 \\
\hline & $\%$ del total & $31.5 \%$ & $29.5 \%$ & $17.1 \%$ & $12.3 \%$ & $9.6 \%$ & $100.0 \%$ \\
\hline
\end{tabular}

A continuación, se presenta la información sobre las competencias desde el punto de vista de los colaboradores. Para ver la importancia de las respuestas, se analizan las medias, las medias más altas indican una mayor importancia a la adquisición de esas competencias.

Tabla No.5

Medias de las Competencia que desean desarrollar los Colaboradores

\begin{tabular}{|c|c|c|c|c|c|c|c|c|}
\hline \multicolumn{9}{|c|}{ Resúmenes de casos } \\
\hline & $\begin{array}{l}\text { Competencia } \\
\text { Comunicación }\end{array}$ & $\begin{array}{c}\text { Competencia } \\
\text { Trabajo en } \\
\text { Equipo }\end{array}$ & $\begin{array}{c}\text { Competencia } \\
\text { Liderazgo }\end{array}$ & $\begin{array}{c}\text { Competencia } \\
\text { Toma de } \\
\text { decisiones }\end{array}$ & $\begin{array}{l}\text { Competencia } \\
\text { Motivación }\end{array}$ & $\begin{array}{c}\text { Competencia } \\
\text { Ética }\end{array}$ & $\begin{array}{l}\text { Competencia } \\
\text { Negociación }\end{array}$ & $\begin{array}{c}\text { Competencia } \\
\text { Relaciones } \\
\text { Interpersonales }\end{array}$ \\
\hline $\mathrm{N}$ & 636 & 636 & 636 & 636 & 636 & 636 & 636 & 636 \\
\hline Media & 3.68 & 3.66 & 3.59 & 3.54 & 3.64 & 3.58 & 3.43 & 3.47 \\
\hline
\end{tabular}

Se observa en la Tabla No. 6, que las competencias que desean mayormente desarrollar los colaboradores son:

- La comunicación

- Trabajo en equipo

- Motivación

- Liderazgo

A continuación, se presenta la información sobre las competencias que se deben desarrollar, según los gerentes. 
Invest. pens. crit. (ISSN 1812-3864)

Vol. 6, No. 1, enero-abril 2018

pp. $36-50$

Tabla No.6

Medias de las Competencia que desean desarrollar los gerentes en los colaboradores

\begin{tabular}{|r|r|r|r|r|r|r|r|r|}
\hline & & & & & & & \\
Competencia \\
& & & & & & & \\
Relaciones \\
\\
& Competencia & CompetenciaTr & Competencia & Competencia & Competencia & Competencia & Competencia \\
Comunicación & abajo en Grupo & Liderazgo & Decisión & Motivación & Ética & Negociación & Interperson. \\
\hline $\mathrm{N}$ & 146 & 146 & 146 & 146 & 146 & 146 & 146 & 146 \\
Media & 3.25 & 3.23 & 3.24 & 3.21 & 3.15 & 3.26 & 3.14 & 3.26 \\
\hline
\end{tabular}

Los Gerentes consideran que las competencias que más se deben desarrollar son:

- Ética

- Relaciones Interpersonales

- Comunicación

- Liderazgo

A continuación, se presenta la información de las necesidades de capacitación de los colaboradores encuestados. Se calculó la media de las respuestas, a una media mayor indica mayor la importancia del curso para capacitación. Ya que la importancia mayor es con respuesta de 5 o 4. Se señalan en rojo las medias más altas.

De los cálculos que se presentan en la Tabla No.7, se concluye que los cursos que más demandan son:

- $\quad$ Habilidad de toma de decisiones

- $\quad$ Planeación Estratégica

- Trabajo en equipo

- Motivación

- Comunicaciones Interpersonales

- $\quad$ Liderazgo

- Supervisión

- Logística

- $\quad$ Solución de Conflictos

- Herramientas Tecnológicas

- Prevención de Accidentes

- $\quad$ Higiene y Salud Ocupacional. 
Invest. pens. crit. (ISSN 1812-3864)

Vol. 6, No. 1, enero-abril 2018

pp. $36-50$

Tabla No.7

Medias de las respuestas hacia la importancia de los cursos

\begin{tabular}{|c|c|c|}
\hline & $\mathrm{N}$ & Media \\
\hline Mercadeo & 636 & 3.58 \\
\hline Ventas & 636 & 3.61 \\
\hline Habilidad de toma de decisiones. & 636 & 3.79 \\
\hline Redacción de Informes & 636 & 3.57 \\
\hline Planeación Estratégica & 636 & 3.71 \\
\hline Trabajo en equipo & 636 & 3.87 \\
\hline Motivación & 636 & 3.85 \\
\hline Contabilidad Administrativa & 636 & 3.64 \\
\hline Comunicaciones Interpersonales & 636 & 3.71 \\
\hline Liderazgo & 636 & 3.80 \\
\hline Supervisión & 636 & 3.70 \\
\hline Logística & 636 & 3.58 \\
\hline Solución de Conflictos & 636 & 3.78 \\
\hline Contabilidad General & 636 & 3.38 \\
\hline Contabilidad Fiscal & 636 & 3.29 \\
\hline Finanzas Generales & 636 & 3.37 \\
\hline Análisis de Estados Financieros. & 636 & 3.38 \\
\hline Presupuesto y Costos & 636 & 3.52 \\
\hline Procesos de Calidad & 636 & 3.51 \\
\hline Formación de Instructores & 636 & 3.44 \\
\hline Estadística & 636 & 3.35 \\
\hline Herramientas Tecnológicas & 636 & 3.64 \\
\hline Prevención de Accidentes & 636 & 3.64 \\
\hline Higiene y Salud Ocupacional & 636 & 3.67 \\
\hline Mantenimiento & 636 & 3.48 \\
\hline Investigación & 636 & 3.44 \\
\hline
\end{tabular}

A continuación, se presenta la información sobre las necesidades de capacitación más apremiantes, según los gerentes. 
Invest. pens. crit. (ISSN 1812-3864)

Vol. 6, No. 1, enero-abril 2018

pp. $36-50$

Tabla No.8

Medias de las necesidades de capacitación

\begin{tabular}{|c|c|c|}
\hline & $\mathrm{N}$ & Media \\
\hline Mercadeo & 146 & 3.37 \\
\hline Ventas & 146 & 3.19 \\
\hline Habilidades de toma de decisiones & 146 & 3.30 \\
\hline Publicidad & 146 & 3.33 \\
\hline Alta Dirección & 146 & 3.16 \\
\hline Planeación Estratégica & 146 & 3.27 \\
\hline Trabajo en Equipo & 146 & 3.30 \\
\hline Motivación & 146 & 3.36 \\
\hline Contabilidad Administrativa & 146 & 3.36 \\
\hline Comunicaciones Interpersonales & 146 & 3.39 \\
\hline Liderazgo & 146 & 3.30 \\
\hline Administración de Recursos Humanos & 146 & 3.30 \\
\hline Logística & 146 & 3.27 \\
\hline Conflictos & 146 & 3.35 \\
\hline Contabilidad General & 146 & 3.20 \\
\hline Contabilidad Fiscal & 146 & 3.09 \\
\hline Finanzas Generales & 146 & 3.21 \\
\hline Análisis de. Estados Financieros & 146 & 3.14 \\
\hline Presupuesto y Costos & 146 & 3.17 \\
\hline Procesos de Calidad & 146 & 3.17 \\
\hline Formación de Instructores & 146 & 3.22 \\
\hline Estadística & 146 & 3.26 \\
\hline Herramientas Tecnológicas & 146 & 3.10 \\
\hline Prevención de Accidentes & 146 & 3.09 \\
\hline Higiene y Salud Ocupacional & 146 & 3.32 \\
\hline Mantenimiento & 146 & 3.36 \\
\hline Investigación & 146 & 3.17 \\
\hline Redacción y Escritura & 146 & 3.23 \\
\hline Otra capacitación & 146 & \\
\hline
\end{tabular}

En la Tabla No. 8, se denota la importancia y necesidad de los siguientes cursos:

- Comunicaciones interpersonales

- Mercadeo

- Mantenimiento

- Motivación

- Contabilidad administrativa

- Resolución de conflictos 
Invest. pens. crit. (ISSN 1812-3864)

Vol. 6, No. 1, enero-abril 2018

pp. $36-50$

- Publicidad

- Liderazgo

- Higiene y Salud

- Recursos Humanos

- Trabajo en equipo

En cuanto al horario de la capacitación, en la Figura No.1, se observa que un 42.3\% de los

colaboradores quieren la capacitación en la noche, un 33.6\% quieren en la mañana, y un 24.1\% quieren la opción en la tarde.

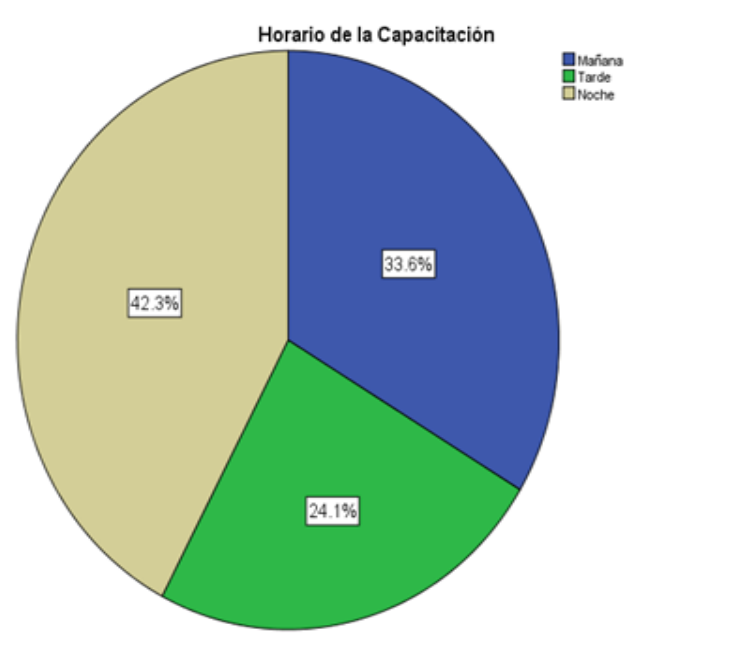

Figura 1: Horario según colaboradores

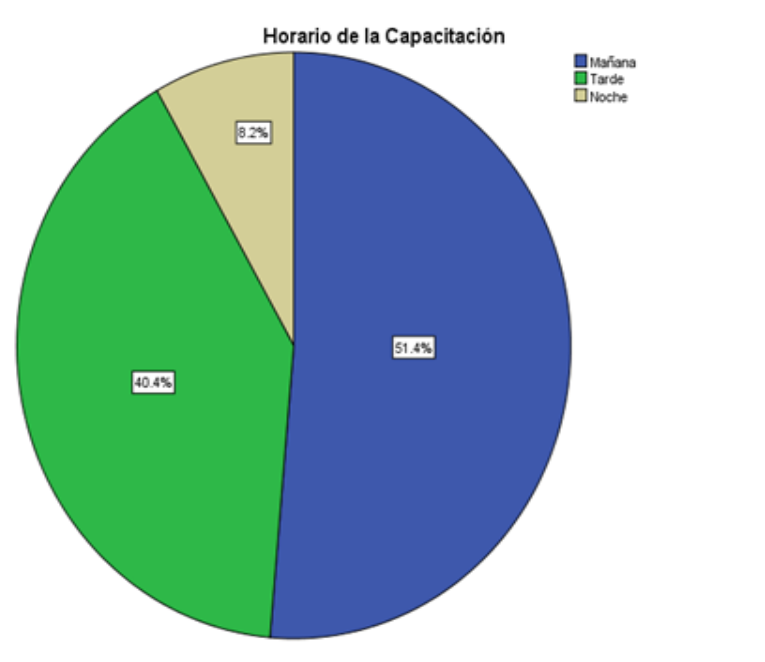

Figura 2: Horario según gerentes

En la Figura No.2, están las opiniones de los gerentes. Un 51.4\% quieren la capacitación en la mañana, un $40.4 \%$ quieren en la tarde, y un $8.2 \%$ quieren la opción en la noche.

En cuanto a la modalidad de estudio según los colaboradores, en la Figura No.3, se observa que un $48.0 \%$ desea modalidad combinada de presencial y virtual, un $32.9 \%$ quieren presencial, y un $19.2 \%$ desean virtual. 
Invest. pens. crit. (ISSN 1812-3864)

Vol. 6, No. 1, enero-abril 2018

pp. 36-50

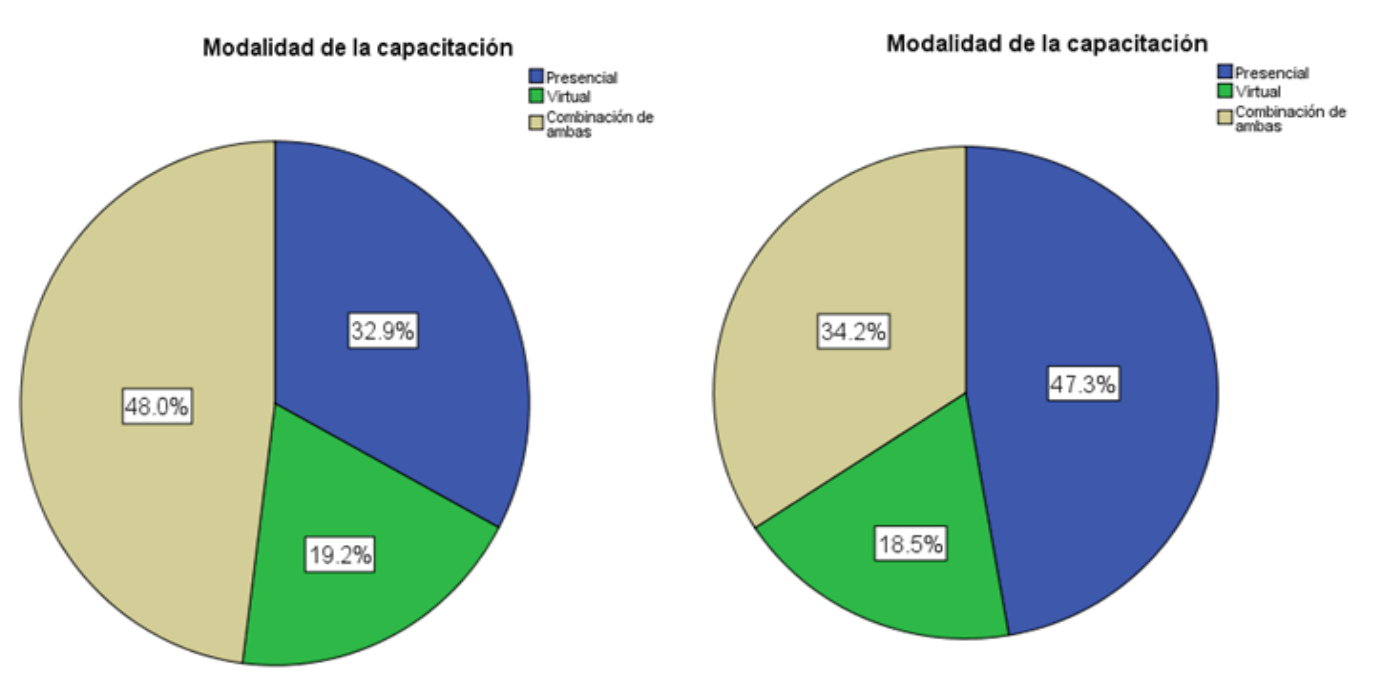

Figura 3: Modalidad según colaboradores

Figura 4: Modalidad según gerentes

En la Figura No.4, vemos la modalidad de estudio según los gerentes. En un 47.3\% desea modalidad presencial, un $34.2 \%$ una combinación de presencial y virtual, y un 18.5\% desean virtual.

En cuanto a los días de capacitación según los colaboradores, en la Figura No.5, se observa que un $60.7 \%$ desean que sean días de semana, un 39.3\% desean en días sábados.

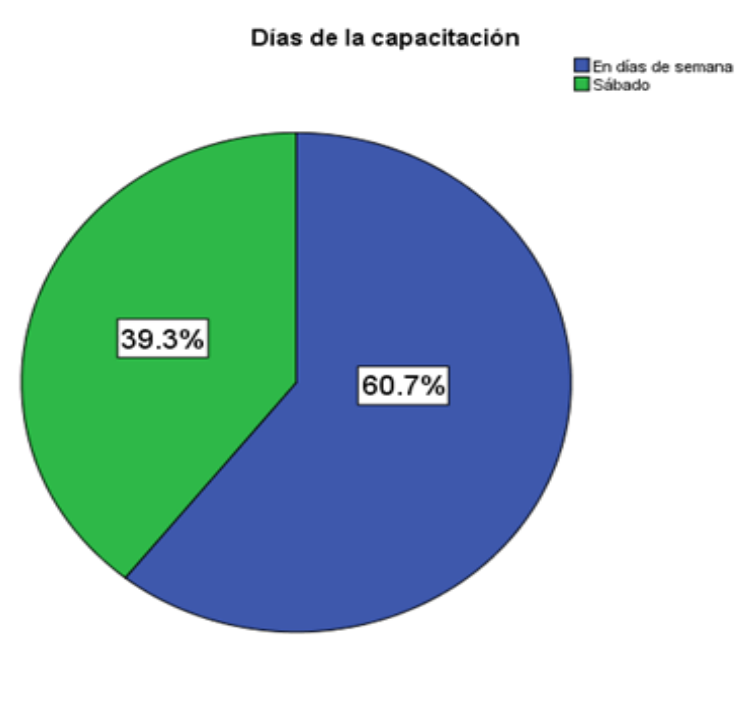

Figura 5: Días de capacitación según colaboradores

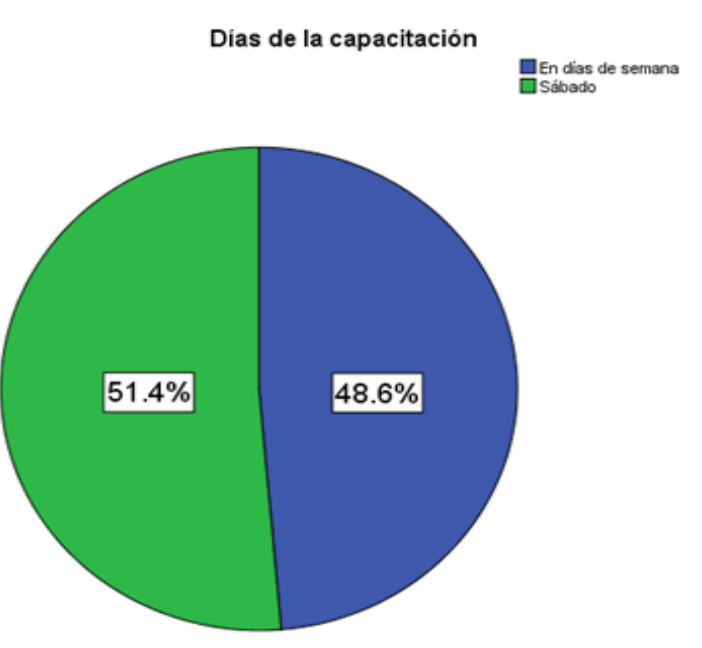

Figura 6: Días de capacitación según gerentes 
Invest. pens. crit. (ISSN 1812-3864)

Vol. 6, No. 1, enero-abril 2018

pp. $36-50$

En la Figura No.6, vemos los días que desean de capacitación según los gerentes. En un 51.4\% desean que sean en sábado, un 48.6\% desean en días de semana.

En cuanto al lugar de capacitación según los colaboradores, en la Figura No.7, se observa que un $57.5 \%$ desean que sea en la misma empresa, un $41.8 \%$ desean que sea en un lugar externo, y un $0.7 \%$ desean que sea en una universidad.

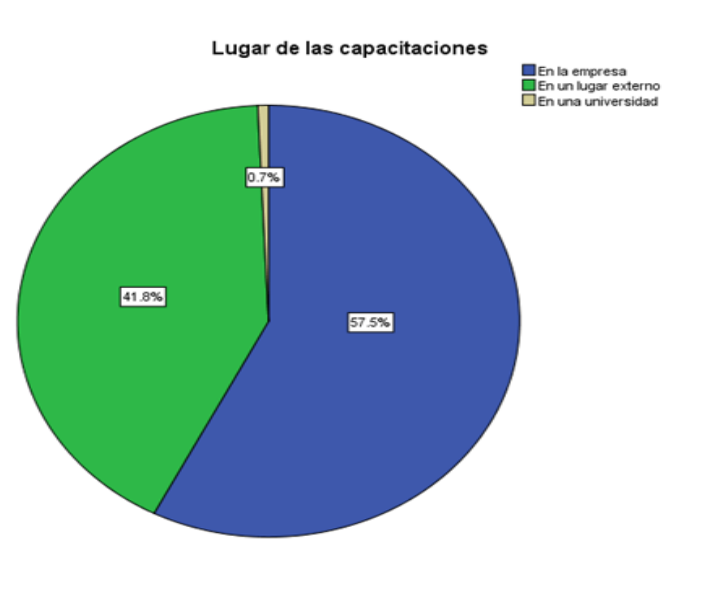

Figura 7: Lugar de capacitación según colaboradores

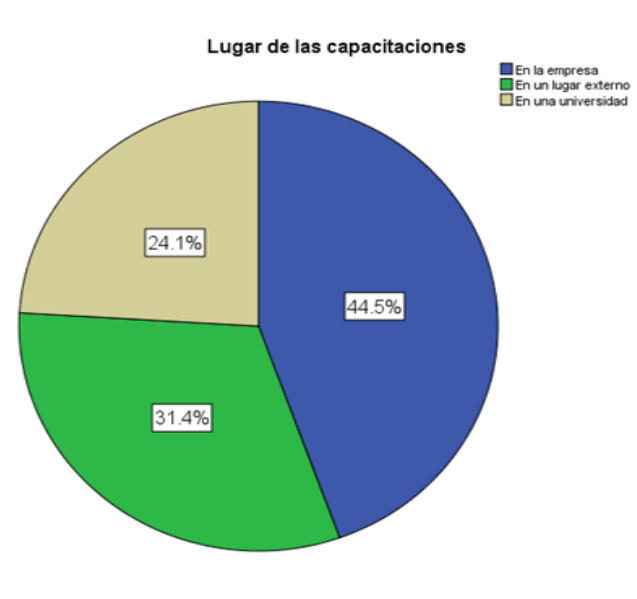

Figura 8: Lugar de capacitación según gerentes

En la Figura No.8, vemos que los gerentes en un 44.5\% desean que sea en la misma empresa, un $31.4 \%$ desean que sea en un lugar externo, y un $24.1 \%$ desean que sea en una universidad. En ambos grupos predomina la respuesta de se haga en la misma empresa.

\section{Conclusiones y recomendaciones}

\section{Conclusiones}

1. Se encuestó a un total de 636 colaboradores en la Ciudad de Panamá. De ese total, unos 251 $(39 \%)$ estudiantes tenían estudios de licenciatura como respuesta predominante, con proporciones similares por sexo. Con respecto a los Gerentes, el 50\% (73 personas) tenían títulos de licenciatura como respuesta predominante, y con proporciones equilibradas por sexo. Además, el 28.8\% (42 empresas) dicen que tienen de 21 a 100 empleados, esta fue la respuesta predominante.

2. El área que más necesita de forma urgente la capacitación, según los colaboradores, es el área administrativa, en segunda opción son operativos. En cuanto a los gerentes opinan que es el área operativa, seguida por el área administrativa.

3. Desde el punto de vista de los colaboradores, ellos piensan que las competencias que desean mayormente desarrollar son: La comunicación, Trabajo en equipo, Motivación y liderazgo. Los 
Invest. pens. crit. (ISSN 1812-3864)

Vol. 6, No. 1, enero-abril 2018

pp. $36-50$

Gerentes consideran que las competencias que más se deben desarrollar son: Ética, Relaciones Interpersonales, Comunicación y Liderazgo

4. Los cursos que más demandan los colaboradores son:
a. Capacitación Habilidad de toma de decisiones
b. Capacitación Planeación Estrategia
c. Capacitación Trabajo en equipo
d. Capacitación Motivación
e. Capacitación Comunicaciones Interpersonales
f. Capacitación Liderazgo
g. Capacitación Supervisión
h. Capacitación Logística
i. Capacitación en Resolución de Conflictos
j. Capacitación Herramientas Tecnológicas
k. Capacitación Prevención de Accidentes
1. Capacitación en Higiene y Salud Ocupacional.

5. Los Gerentes manifiestan que los cursos con más importancia y necesidad son los siguientes:
a. Comunicaciones interpersonales
b. Mercadeo
c. Mantenimiento
d. Motivación
e. Contabilidad administrativa
f. Resolución de conflictos
g. Publicidad
h. Liderazgo
i. Higiene y Salud Ocupacional
j. Recursos Humanos
k. Trabajo en equipo

6. En cuanto al horario, los colaboradores en una mayor cantidad (269) les gusta el horario de noche, seguido por mañana (214), y en tercera opción en la tarde. En cuanto a qué horario considera se debe desarrollar la capacitación, un 51.4\% opinan que, en la mañana, un 40.4\% opinan que, en la tarde, y solo un $8.2 \%$ dicen que en la noche.

7. La mayor cantidad, 386 personas desean que la capacitación sea en días de semana, y unas 250 personas desean que sea sábado. En cuanto a qué día considera se debe desarrollar la capacitación, un 51.4\% consideran que se debe ofrecer los sábados, y un $48.6 \%$ dicen que en los días de las semanas. 
Invest. pens. crit. (ISSN 1812-3864)

Vol. 6, No. 1, enero-abril 2018

pp. $36-50$

8. Un total de 283 colaboradores desean que las capacitaciones sean en las empresas, unos 200 colaboradores desean que sea en un lugar externo, y unos 153 colaboradores desean que sea en una universidad. En cuanto a los gerentes, un 57.5\% desean que, sea la empresa, un 41.8\% opinan que, en un lugar externo, y sólo un $0.7 \%$ dicen que en una universidad.

\section{Recomendaciones}

1. Que la USMA a través de la Dirección de Educación Continuada, haga una oferta a las empresas, ofertando los cursos que señalan los colaboradores y gerentes como cursos más necesitados en las empresas, y que se tome en consideración el desarrollo de los cursos en las empresas, porque ambos grupos así lo han señalado como preferencia.

2. Que la USMA haga una difusión de los cursos que se ofertan, y los horarios establecidos.

3. Que las Facultades de la USMA continúen realizando investigaciones en diversos sectores para valorar las necesidades de formación específicas en los diversos sectores económicos.

4. Que la USMA, desarrolle nuevas carreras acordes a los cambios actuales, y desarrolle un doctorado en el área empresarial, para dar continuidad a los estudios de maestrías, ya que hay poca oferta y es una necesidad para el desarrollo del país.

5. Que la universidad realice convenios con gremios y empresas, para potenciar la capacitación en las empresas, y ofertar carreras de postgrados a instituciones del Estado y al sector empresarial.

\section{Agradecimiento}

A todos los estudiantes de la USMA, que apoyaron en la aplicación de las encuestas, y en la captación de los datos. A los profesores que brindaron la orientación y guía a los estudiantes, y al Decano Eduardo Pazmiño, por el apoyo brindado.

\section{Bibliografía}

1. HERNÁNDEZ, ROBERTO, CARLOS FERNÁNDEZ Y PILAR BAPTISTA. (2014). Metodología de la Investigación. 6ta edición. Editora McGraw-Hill, México.

2. MEJÍA GIRALDO, ARMANDO Y ARTURO MONTOYA SERRANO. (2013). Capacitación integral del talento humano por competencias, orientada hacia la innovación tecnológica, Edición: Primera, Editorial: Universidad de San Buenaventura Seccional Cali, Colombia. 
Invest. pens. crit. (ISSN 1812-3864)

Vol. 6, No. 1, enero-abril 2018

pp. $36-50$

3. RITA DEL CARMEN CASTILLO CONTRERAS. (2012). Desarrollo del capital humano en las organizaciones Red Tercer Milenio, México.

4. DE LA CALLE DURÁN /MARÍA DEL CARMEN Y MARTA ORTIZ-DE-URBINA CRIADO. (2014). Fundamentos de Recursos Humanos, Editora Pearson, España.

5. PINTO ROBERTO. (2012). Planeación estratégica de capacitación empresarial, Editora McGraw Hill, México.

6. DUNNING, DONNA. (2012). Capacitación, Dirección y Asesoramiento en el trabajo, Editora CECSA, México.

7. PÉREZ, CESAR. (2014). Técnicas Estadísticas con SPSS. Editora Prentice-Hall, España.

8. Manual del usuario del sistema básico de IBM SPSS Statistics 24., 2016. Estados Unidos de América. 